\title{
Analysis of differentially expressed genes in various stages of Duchenne muscular dystrophy by using a network view
}

\author{
D. Wang, Z.J. Wang, X.X. Song, L.H. Pu, X. Li and Y. Wang \\ Department of Neurology, Xi'an Children's Hospital, Xi'an, Shaanxi, China \\ Corresponding author: D. Wang \\ E-mail: wangdong6928@yahoo.cn
}

Genet. Mol. Res. 12 (4): 4480-4488 (2013)

Received February 20, 2013

Accepted August 18, 2013

Published October 10, 2013

DOI http://dx.doi.org/10.4238/2013.October.10.13

\begin{abstract}
Duchenne muscular dystrophy (DMD), which is caused by mutations in the $\mathrm{X}$-linked dystrophin gene, is a severe and progressive neuromuscular disease with no available cure. By integrating 2 microarray datasets from the Gene Expression Omnibus, we identified differentially expressed genes in 2 stages of DMD and systematically explored their potential disease-related mechanisms using a network view. Twenty differentially expressed genes were detected in various stages of DMD. According to the network with dystrophin as its center, none of the 20 proteins interacts with dystrophin directly. IQ motif-containing GTPaseactivating protein 1 was found in the 2nd-level neighbors with a degree of 21. Microtubule-associated protein tau, membrane metallo-endopeptidase, interleukin 13 receptor alpha 1 , and multiple epidermal growth factor-like domains 6 were found in the 3rd-level neighbors. These identifications require further investigation, as this report is the first of possible associations between DMD and these proteins. Analysis of differentially expressed genes through this network view may provide important information for further exploration of underlying mechanisms of DMD.
\end{abstract}

Key words: Duchenne muscular dystrophy; Differential expression; Protein-protein interaction network 


\section{INTRODUCTION}

Duchenne muscular dystrophy (DMD), which affects 1 in 3500 newborn boys (Emery, 2002), is a severe and progressive neuromuscular disease caused by mutations in the X-linked dystrophin gene. Absence or defect of the protein dystrophin disrupts its essential function of connecting the subsarcolemmal cytoskeleton to the sarcolemma, resulting in progressive muscle degeneration. Typically, patients without intervention lose independent ambulation by the age of 13 years (Hoffman et al., 1987) and experience cardiac or respiratory failure in their mid- to late 20s, with a mean age of death of approximately 19 years (Bushby et al., 2010a). Cognitive dysfunction might also be present (Anderson et al., 2002). Symptoms of DMD are usually initially observed between the ages of 2 and 5 years (Dubowitz, 1978; Jennekens et al., 1991), although elevated serum creatine kinase level and abnormal muscle histology are always present in patients. Thus, the first 2 years are considered clinically presymptomatic (Pescatori et al., 2007).

Since the genetic etiology of DMD was well analyzed over 20 years ago (Koenig et al., 1987; Kunkel et al., 1987), numerous therapeutic strategies have been proposed, including gene-based therapy, cell-based therapy, and pharmacological agents. Multidisciplinary care has also been recommended (Bushby et al., 2010b). However, these approaches have many hurdles yet to overcome, and no cure for DMD is presently available. Understanding the pathomechanisms for DMD may lead to alternatives to currently inadequate therapeutic strategies. Previous studies have shown that DMD patients exhibit symptoms related to various proteins or molecules, such as growth factors (Gehrig et al., 2012), nitric oxide synthase (Altamirano et al., 2012), phospholipase (Lindahl et al., 1995), and cytokines (De Pasquale et al., 2012), suggesting that "molecule groups" instead of single or a few proteins should be explored in the investigation of the DMD pathomechanism. Protein-protein interaction (PPI) is crucial for all biological processes (Stelzl et al, 2005) because the majority of proteins function with other proteins. Therefore, biological processes should be considered complex PPI networks of interconnected proteins. In other words, analysis from a network view would provide a better understanding of the mechanism underlying DMD progression.

High-throughput experimental strategies enable identification of candidate gene sets associated with the symptoms of DMD patients. However, few of these efforts have focused on the network downstream of dystrophin, which contributes to development and progression from the presymptomatic phase (PP) of the disease to the symptomatic phase (SP) and represents the pathomechanisms of DMD. Knowledge of these networks would facilitate understanding of the molecular mechanisms of DMD.

Our hypothesis is that dysfunctional genes/proteins and their interactions are strongly related to specific states of the disease and can capture the pathogenic characteristics of corresponding disease stages. Differentially expressed genes between SP and PP may reflect central elements in the progression of DMD from PP to SP. Integrating microarray data from both PP and SP, we identified differentially expressed genes/proteins between SP and PP and carried out biological network analysis for their interactions with the DMD protein to get a better understanding of the molecular mechanism underlying DMD progression.

\section{MATERIAL AND METHODS}

\section{Microarray data}

Two datasets (GSE6011 and GSE300741) were downloaded from the Gene Expres- 
sion Omnibus (http://www.ncbi.nlm.nih.gov/geo/) database. Sample information for 19 presymptomatic samples, 8 symptomatic samples, and 14 normal samples is listed in Table 1. Both datasets were based on the GPL96 platform: [HG-U133A] Affymetrix Human Genome U133A Array.

Table 1. Characteristics of the samples.

\begin{tabular}{|c|c|c|c|c|c|}
\hline GEO accession & ID & Phase & Age (years) & Gender & Dystrophin expression \\
\hline GSM139515 & PP01 & Presymptomatic & 0.1 & $\mathrm{M}$ & Protein absent; mRNA normal \\
\hline GSM139516 & PP02 & Presymptomatic & 0.2 & M & Protein absent; mRNA reduced \\
\hline GSM139517 & PP03 & Presymptomatic & 0.3 & M & Protein absent; mRNA reduced \\
\hline GSM139519 & PP04 & Presymptomatic & 0.4 & M & Protein absent; mRNA reduced \\
\hline GSM139520 & PP05 & Presymptomatic & 0.4 & M & Protein absent; mRNA reduced \\
\hline GSM139521 & PP06 & Presymptomatic & 0.5 & M & Protein absent; mRNA weakly reduced \\
\hline GSM139522 & PP07 & Presymptomatic & 0.5 & M & Protein absent; mRNA reduced \\
\hline GSM139523 & PP08 & Presymptomatic & 0.6 & M & Protein absent; mRNA reduced \\
\hline GSM139524 & PP09 & Presymptomatic & 0.7 & M & Protein absent; mRNA reduced \\
\hline GSM139525 & PP10 & Presymptomatic & 0.7 & M & Protein absent; mRNA reduced \\
\hline GSM139526 & PP11 & Presymptomatic & 0.9 & M & Protein absent; mRNA normal \\
\hline GSM139527 & PP12 & Presymptomatic & 1.0 & M & Protein absent; mRNA reduced \\
\hline GSM139528 & PP13 & Presymptomatic & 1.0 & M & Protein absent; mRNA reduced \\
\hline GSM139529 & PP14 & Presymptomatic & 1.2 & M & Protein absent; mRNA reduced \\
\hline GSM139530 & PP15 & Presymptomatic & 1.2 & M & Protein absent; mRNA reduced \\
\hline GSM139531 & PP16 & Presymptomatic & 1.3 & M & Protein absent; mRNA reduced \\
\hline GSM139532 & PP17 & Presymptomatic & 1.7 & M & Protein absent; mRNA reduced \\
\hline GSM139533 & PP18 & Presymptomatic & 1.7 & M & Protein absent; mRNA reduced \\
\hline GSM139534 & PP19 & Presymptomatic & 1.8 & M & Protein absent; mRNA reduced \\
\hline GSM121357 & SP20 & Symptomatic & 5.0 & M & Protein absent \\
\hline GSM121361 & SP21 & Symptomatic & 8.0 & M & Protein absent \\
\hline GSM121363 & SP22 & Symptomatic & 7.0 & M & Protein absent \\
\hline GSM121368 & SP23 & Symptomatic & 8.0 & M & Protein absent \\
\hline GSM121369 & SP24 & Symptomatic & 7.0 & M & Protein absent \\
\hline GSM139535 & SP25 & Symptomatic & 2.3 & M & Protein absent; mRNA reduced \\
\hline GSM139536 & SP26 & Symptomatic & 3.9 & M & Protein absent; mRNA reduced \\
\hline GSM139537 & SP27 & Symptomatic & 5.1 & M & Protein absent; mRNA reduced \\
\hline GSM139501 & $\mathrm{C} 28$ & Control & 0.4 & M & Normal \\
\hline GSM139502 & $\mathrm{C} 29$ & Control & 0.5 & $\mathrm{~F}$ & Normal \\
\hline GSM139503 & $\mathrm{C} 30$ & Control & 0.5 & $\mathrm{~F}$ & Normal \\
\hline GSM139504 & $\mathrm{C} 31$ & Control & 0.5 & $\mathrm{M}$ & Normal \\
\hline GSM139505 & $\mathrm{C} 32$ & Control & 0.5 & M & Normal \\
\hline GSM139506 & $\mathrm{C} 33$ & Control & 0.6 & M & Normal \\
\hline GSM139507 & C34 & Control & 0.7 & $\mathrm{~F}$ & Normal \\
\hline GSM139508 & $\mathrm{C} 35$ & Control & 0.9 & M & Normal \\
\hline GSM139509 & $\mathrm{C} 36$ & Control & 1.5 & M & Normal \\
\hline GSM139510 & $\mathrm{C} 37$ & Control & 2.8 & M & Normal \\
\hline GSM139511 & C38 & Control & 3.0 & M & Normal \\
\hline GSM139512 & C39 & Control & 4.2 & M & Normal \\
\hline GSM139513 & $\mathrm{C} 40$ & Control & 5.0 & M & Normal \\
\hline GSM139514 & $\mathrm{C} 41$ & Control & 9.0 & M & Normal \\
\hline
\end{tabular}

\section{Detection of differentially expressed genes}

Entire data sets including CEL- and SOFT-formatted family files for the 41 samples from the two datasets (GSE6011 and GSE300741) were downloaded. Raw data from the CEL files, which were generated from satisfactory image files, were normalized via robust multiarray analysis (Irizarry et al., 2003) following 3 steps: first, background noise effects and processing artifacts were neutralized with model-based background correction. Second, quantile normalization was used to align expression values to a common scale. Third, data were summarized and an iterative median polishing procedure was used to generate a single expression 
value for each probe set. The resulting log2-transformed robust multi-array analysis expression value was derived through probe set-level analysis from the raw CEL files.

Statistical $t$-tests with multiple test correction using the Benjamini and Hochberg procedure (Benjamini and Hochberg, 1995) were carried out for the symptomatic-presymptomatic pairs to detect differentially expressed genes with the threshold of significantly expressed genes set at 0.01 . The differentially expressed genes were detected as follows: First, data from the symptomatic-presymptomatic pair of patients were used to detect differentially expressed genes. Second, data from controls were divided into 2 subsets according to age (older than 2 years and younger than 2 years). Normal age-related differentially expressed genes were detected in these sets. Finally, differentially expressed genes correlated with DMD progression were finalized as those detected in the symptomatic-presymptomatic patients pair but not identified in the control pair. Up- or down regulation of differentially expressed genes were determined with fold change. All of these procedures were carried out using R statistical software (v2.14.1) with BioConductor, limma packages (3.12.1), and libraries (Smyth et al., 2005).

\section{PPI network analysis}

We used Cytoscape (V 2.8.3; http://www.cytoscape.org/) and the Human Protein Reference Database (release date, 2009) (Keshava Prasad et al., 2009) to construct the network related to the DMD protein. Up to 3rd-level neighbors were allowed. First-level neighbors included proteins that interacted with the DMD protein directly. Second-level neighbors included proteins that interacted with 1st-level neighbors directly, whereas 3rd-level neighbors included proteins that interacted with 2nd-level neighbors directly. Differentially expressed proteins correlated with DMD progression identified as described above were checked to confirm their interaction with the DMD protein to characterize the PPI network changes that occurred with DMD progression.

\section{RESULTS}

Of the well-characterized human genes in the Affymetrix Human Genome U133A Array, 28 genes were found to be differentially expressed in the symptomatic-presymptomatic patient pairs. Eight of those genes were excluded because they were also differentially expressed in the 2 control pairs. The 20 remaining genes (Table 2) were considered to play important roles in the progression of DMD. An expression heatmap of the 20 differentially expressed genes in all samples is shown in Figure 1. According to the PPI network analysis, none of the proteins encoded by these 20 genes was found in the 1st-level neighbors of the DMD protein. In the 2 nd-level network, the IQ motif containing GTPase-activating protein 1 (IQGAP1), which had a high degree of 21 , was found. In the 3rd-level network, we found IQGAP1 protein and 4 additional proteins-microtubule-associated protein tau (MAPT), membrane metallo-endopeptidase, interleukin receptor 13 alpha 1, and multiple epidermal growth factor-like domains 6-with degrees of 7, 2, 1, and 1, respectively. Degree is the simplest topological index, corresponding to the number of proteins or nodes directly connected to a given node or protein. Proteins with higher degrees are connected to more proteins and tend to play important roles in body development and metabolism. Illustrations of the 2nd- and 3rd-level PPI networks are shown in Figures 2 and 3, respectively. 
Table 2. Identified genes contributes to the progression of Duchenne muscular dystrophy.

\begin{tabular}{llr}
\hline Symbol & Full name & P \\
\hline ARHGAP32 & Rho GTPase activating protein 32 & $1.92 \mathrm{E}-03$ \\
CCDC69 & Coiled-coil domain containing 69 & $5.41 \mathrm{E}-03$ \\
CRTAP & Cartilage associated protein & $1.76 \mathrm{E}-04$ \\
FAM149A & Family with sequence similarity 149, member A & $1.98 \mathrm{E}-03$ \\
FRZB & Frizzled-related protein & $1.02 \mathrm{E}-03$ \\
IL13RA1 & Interleukin 13 receptor, alpha 1 & $7.52 \mathrm{E}-03$ \\
IQGAP1 & IQ motif containing GTPase activating protein 1 \\
KCTD9 & Potassium channel tetramerisation domain containing 9 & $7.72 \mathrm{E}-03$ \\
MAPT & Microtubule-associated protein tau & $9.94 \mathrm{E}-03$ \\
MEGF6 & Multiple EGF-like-domains 6 & $1.79 \mathrm{E}-03$ \\
MKKS & McKusick-Kaufman syndrome & $4.01 \mathrm{E}-03$ \\
MME & Membrane metallo-endopeptidase & $6.82 \mathrm{E}-03$ \\
MOSPD1 & Motile sperm domain containing 1 & $1.09 \mathrm{E}-03$ \\
NPY6R & Neuropeptide Y receptor Y6 (pseudogene) & $1.79 \mathrm{E}-03$ \\
OXCT1 & 3-oxoacid CoA-transferase 1 & $7.52 \mathrm{E}-03$ \\
PHF20 & PHD finger protein 20 & $7.39 \mathrm{E}-03$ \\
PMS2P1 & Postmeiotic segregation increased 2 pseudogene 1 & $6.73 \mathrm{E}-03$ \\
SLC15A2 & Solute carrier family 15 (H+/peptide transporter), member 2 & $7.53 \mathrm{E}-03$ \\
SSPN & Sarcospan (Kras oncogene-associated gene) & $5.14 \mathrm{E}-03$ \\
WISP2 & WNT1 inducible signaling pathway protein 2 & $7.39 \mathrm{E}-03$ \\
\hline
\end{tabular}

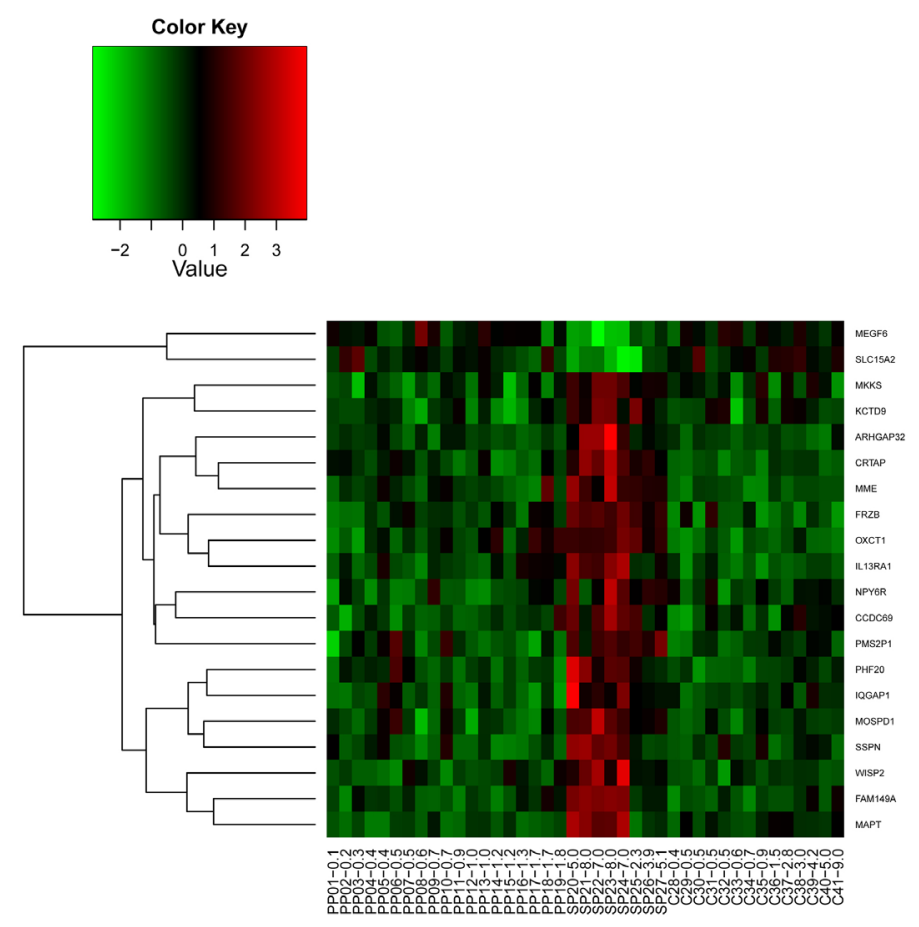

Figure 1. Heatmap for the expression of the 20 genes selected. Information for each sample corresponding to Table 1 are shown in the $\mathrm{X}$ axis format as: sample id-age. The 20 differentially expressed genes were combined and then hierarchically clustered to represent the expression patterns with average linkage and Euclidean distance as a measurement of similarity. The expression values were log ratios normalized according to the procedure described in the Methods section. Red and green represent upregulation and downregulation, respectively. Precise color scheme is illustrated in the color key. 


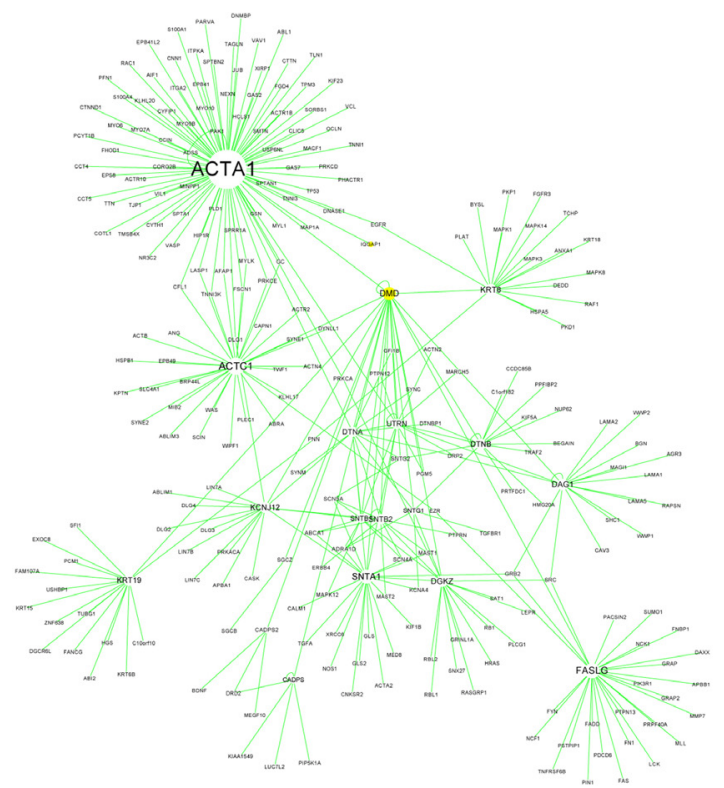

Figure 2. Second-level PPI network for dystrophin. First-level proteins refer to proteins interact with dystrophin directly. Second-level proteins refer to those interact with first-level proteins. This figure illustrate the interaction network for dystrophin, including proteins up to the second level.

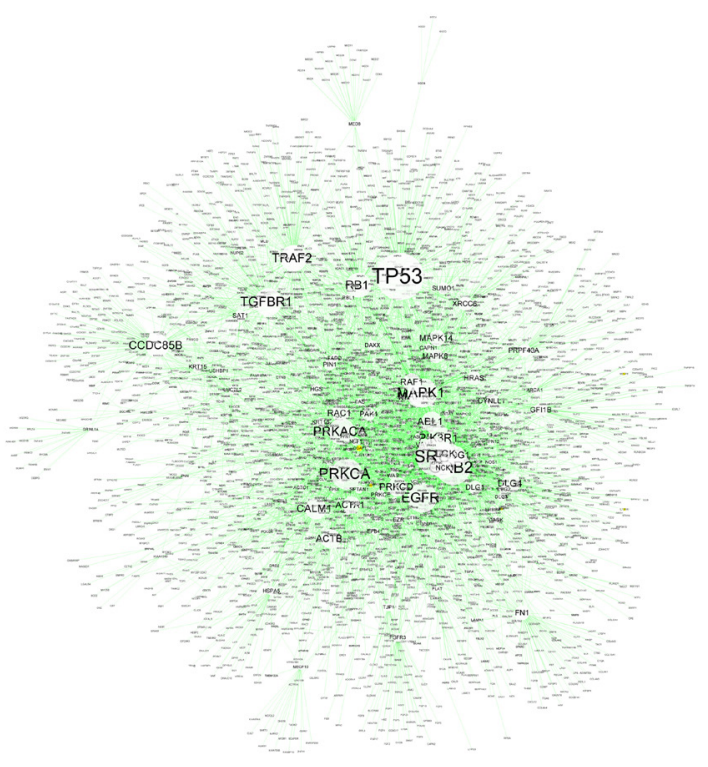

Figure 3. Third-level PPI network for dystrophin. First-level proteins refer to proteins interact with dystrophin directly. Second-level proteins refer to those interact with first-level proteins and third-level proteins refer to those interact with second-level proteins. This figure illustrates the interaction network for dystrophin, including proteins up to the third level. 


\section{DISCUSSION}

The pathophysiology of DMD involves many secondary changes (Gorospe et al., 1994; Chen et al., 2000; Head, 2010; Altamirano et al., 2012; De Pasquale et al., 2012; Gehrig et al., 2012) owing to mutation in the dystrophin gene. This high complex progression of DMD presents challenges for curing the disease. Understanding the pathomechanisms for DMD from a network view may improve understanding of DMD and contribute potential targets for new therapeutic strategies because most proteins function with other proteins. Herein we detected differentially expressed genes that contribute to DMD progression using 2 data sets from the Gene Expression Omnibus database and characterized their interaction with the DMD protein using a network view.

As shown in Figure 1, 20 genes with differential expression only in SP and PP patient pairs were identified as genes contributing to the progression of DMD. As expected, the DMD gene did not show differential expression between the 2 phases because the DMD mutation exists even when patients are phenotypically indistinguishable from normal individuals. According to the network analysis, none of the proteins encoded by these 20 genes interacts with dystrophin directly, indicating that the biological function of the proteins that interact directly with dystrophin do not change substantially in the progression of DMD.

One of the identified differentially expressed genes encodes IQGAP1, which belongs to the 2nd-level neighbors of DMD and has a high degree of 21. No report about the relationship between IQGAP1 and DMD has been published until now. As a scaffolding protein, IQGAP1 binds directly to an impressive collection of other proteins (Mateer et al., 2003), including $\mathrm{F}$-actin (or alpha actins encoded by ACTA1 in skeletal muscle), which interacts directly with dystrophin. F-actin is polymerized from G-actin. A previous study (Prins et al., 2008) has shown that skeletal muscle-specific ablation of G-actin does not exacerbate the dystrophin-deficient phenotype in mice. Thus, the relationship with IQGAP1 and deterioration of DMD may not involve the dysfunction of actins. IQGAP1 may affect the progression of DMD through its interaction with $\mathrm{Ca}^{2+}$ /calmodulin (Joyal et al., 1997; Ho et al., 1999) predominantly via its 4 IQ motifs, because $\mathrm{Ca}^{2+}$ and calmodulin modulate numerous cellular functions, including muscle contraction (Berridge et al., 2000). A previous study (Weissbach et al., 1998) has shown that the interaction of IQGAP1 and myosin essential light chain have significant consequences for actomyosin contractility, suggesting another possible mechanism for IQGAP1 in the progression of DMD.

Protein encoded by a gene called MAPT was found in the 3rd-level neighbors of dystrophin with a degree of 7. MAPT mutations have been associated with several neurodegenerative disorders, such as frontotemporal lobar degeneration (Sieben et al., 2012) and Parkinson disease (Farrer, 2006). Differential expression of MAPT may be related to the cognitive dysfunction of DMD patients; however, further research is needed for confirmation because previous reports have indicated that cognitive dysfunction in DMD patients is non-progressive (Bushby et al., 2010a), whereas the differential expression pattern existed between SP and PP in our study. Membrane metallo-endopeptidase was also found in the 3rd-level neighbors with a degree of 2. Its differential expression may contribute to the psychological state changes of the patients that accompany the progression of DMD because the function of this protein is related to the inactivation of enkephalins, which have been implicated in the regulation of mood, anxiety, reward, euphoria, and pain (Comings et al., 2000). 
Among the other proteins that do not interact with dystrophin in up to three levels, sarcospan (SSPN) is especially notable. As a unique tetraspanin-like core component of the dystrophin- and utrophin-glycoprotein complexes in skeletal muscle, SSPN and its modulation of integrin signaling are necessary for extracellular matrix attachment and muscle force development (Marshall et al., 2012). Peter et al. (2008) have also reported that to compensate for the loss of dystrophin, SSPN may increase utrophin-glycoprotein complex levels at the extrasynaptic membrane to stabilize the sarcolemma. Thus, SSPN is worthy of further investigation and may be a potential target for DMD treatment.

In summary, we analyzed differentially expressed genes in the progression of DMD using a network view. The genes identified herein may help to understand the symptoms and especially the progression of DMD and at the same time spur new directions for therapeutic interventions, which are at present inadequate.

\section{REFERENCES}

Altamirano F, Lopez JR, Henriquez C, Molinski T, et al. (2012). Increased resting intracellular calcium modulates NFkappaB-dependent inducible nitric-oxide synthase gene expression in dystrophic mdx skeletal myotubes. J. Biol. Chem. 287: 20876-20887.

Anderson JL, Head SI, Rae C and Morley JW (2002). Brain function in Duchenne muscular dystrophy. Brain 125: 4-13.

Benjamini Y and Hochberg Y (1995). Controlling the false discovery rate: A practical and powerful approach to multiple testing. J. Roy. Stat. Soc. S B Met. 57: 289-300.

Berridge MJ, Lipp P and Bootman MD (2000). The versatility and universality of calcium signalling. Nat. Rev. Mol. Cell Biol. 1: 11-21.

Bushby K, Finkel R, Birnkrant DJ, Case LE, et al. (2010a). Diagnosis and management of Duchenne muscular dystrophy, part 1: diagnosis, and pharmacological and psychosocial management. Lancet Neurol. 9: 77-93.

Bushby K, Finkel R, Birnkrant DJ, Case LE, et al. (2010b). Diagnosis and management of Duchenne muscular dystrophy, part 2: implementation of multidisciplinary care. Lancet Neurol. 9: 177-189.

Chen YW, Zhao P, Borup R and Hoffman EP (2000). Expression profiling in the muscular dystrophies: identification of novel aspects of molecular pathophysiology. J. Cell Biol. 151: 1321-1336.

Comings DE, Dietz G, Gade-Andavolu R, Blake H, et al. (2000). Association of the neutral endopeptidase (MME) gene with anxiety. Psychiatr. Genet. 10: 91-94.

De Pasquale L, D'Amico A, Verardo M, Petrini S, et al. (2012). Increased muscle expression of interleukin-17 in Duchenne muscular dystrophy. Neurology 78: 1309-1314.

Dubowitz V (1978). Muscle disorders in childhood. Major Problem. Clin. Pediatr. 16: iii-xiii, 1-282.

Emery AE (2002). The muscular dystrophies. Lancet 359: 687-695.

Farrer MJ (2006). Genetics of Parkinson disease: paradigm shifts and future prospects. Nat. Rev. Genet. 7: 306-318.

Gehrig SM, van der Poel C, Hoeflich A, Naim T, et al. (2012). Therapeutic potential of PEGylated insulin-like growth factor I for skeletal muscle disease evaluated in two murine models of muscular dystrophy. Growth Horm. IGF Res. 22: 69-75.

Gorospe JR, Tharp MD, Hinckley J, Kornegay JN, et al. (1994). A role for mast cells in the progression of Duchenne muscular dystrophy? Correlations in dystrophin-deficient humans, dogs, and mice. J. Neurol. Sci. 122: 44-56.

Head SI (2010). Branched fibres in old dystrophic mdx muscle are associated with mechanical weakening of the sarcolemma, abnormal $\mathrm{Ca}^{2+}$ transients and a breakdown of $\mathrm{Ca}^{2+}$ homeostasis during fatigue. Exp. Physiol. 95: 641-656.

Ho YD, Joyal JL, Li Z and Sacks DB (1999). IQGAP1 integrates $\mathrm{Ca}^{2+} /$ calmodulin and Cdc42 signaling. J. Biol. Chem. 274: 464-470.

Hoffman EP, Brown RH Jr and Kunkel LM (1987). Dystrophin: the protein product of the Duchenne muscular dystrophy locus. Cell 51: 919-928.

Irizarry RA, Hobbs B, Collin F, Beazer-Barclay YD, et al. (2003). Exploration, normalization, and summaries of high density oligonucleotide array probe level data. Biostatistics 4: 249-264.

Jennekens FG, ten Kate LP, de Visser M and Wintzen AR (1991). Diagnostic criteria for Duchenne and Becker muscular dystrophy and myotonic dystrophy. Neuromuscul. Disord. 1: 389-391. 
Joyal JL, Annan RS, Ho YD, Huddleston ME, et al. (1997). Calmodulin modulates the interaction between IQGAP1 and Cdc42. Identification of IQGAP1 by nanoelectrospray tandem mass spectrometry. J. Biol. Chem. 272: 15419-15425.

Keshava Prasad TS, Goel R, Kandasamy K, Keerthikumar S, et al. (2009). Human Protein Reference Database - 2009 update. Nucleic Acids Res. 37: D767-D772.

Koenig M, Hoffman EP, Bertelson CJ, Monaco AP, et al. (1987). Complete cloning of the Duchenne muscular dystrophy (DMD) cDNA and preliminary genomic organization of the DMD gene in normal and affected individuals. Cell 50: 509-517.

Kunkel LM, Monaco AP, Hoffman E, Koenig M, et al. (1987). Molecular studies of progressive muscular dystrophy (Duchenne). Enzyme 38: 72-75.

Lindahl M, Backman E, Henriksson KG, Gorospe JR, et al. (1995). Phospholipase A2 activity in dystrophinopathies. Neuromuscul. Disord. 5: 193-199.

Marshall JL, Chou E, Oh J, Kwok A, et al. (2012). Dystrophin and utrophin expression require sarcospan: loss of alpha7 integrin exacerbates a newly discovered muscle phenotype in sarcospan-null mice. Hum. Mol. Genet. 21: 4378-4393.

Mateer SC, Wang N and Bloom GS (2003). IQGAPs: integrators of the cytoskeleton, cell adhesion machinery, and signaling networks. Cell Motil. Cytoskeleton 55: 147-155.

Pescatori M, Broccolini A, Minetti C, Bertini E, et al. (2007). Gene expression profiling in the early phases of DMD: a constant molecular signature characterizes DMD muscle from early postnatal life throughout disease progression. FASEB J. 21: 1210-1226.

Peter AK, Marshall JL and Crosbie RH (2008). Sarcospan reduces dystrophic pathology: stabilization of the utrophinglycoprotein complex. J. Cell Biol. 183: 419-427.

Prins KW, Lowe DA and Ervasti JM (2008). Skeletal muscle-specific ablation of gamma(cyto)-actin does not exacerbate the mdx phenotype. PLoS One 3: e2419.

Sieben A, Van Langenhove T, Engelborghs S, Martin JJ, et al. (2012). The genetics and neuropathology of frontotemporal lobar degeneration. Acta Neuropathol. 124: 353-372.

Smyth GK, Michaud J and Scott HS (2005). Use of within-array replicate spots for assessing differential expression in microarray experiments. Bioinformatics 21: 2067-2075.

Stelzl U, Worm U, Lalowski M, Haenig C, et al. (2005). A human protein-protein interaction network: a resource for annotating the proteome. Cell 122: 957-968.

Weissbach L, Bernards A and Herion DW (1998). Binding of myosin essential light chain to the cytoskeleton-associated protein IQGAP1. Biochem. Biophys. Res. Commun. 251: 269-276. 